\title{
Biochemical Signatures in Growth Hormone Deficiency: a Pilot Study Using Metabolomics Approaches by Direct Infusion-mass Spectrometry
}

\author{
Breno De San-Martin \\ Escola Paulista de Medicina, Universidade Federal de São Paulo, SP \\ Vinícius Ferreira \\ Instituto de Química de São Carlos, Universidade de São Paulo, SP \\ Mariana Bitencourt \\ Unidade de Endocrinologia do Desenvolvimento, Faculdade de Medicina da Universidade de São Paulo, SP \\ Paulo Cesar Pereira \\ Unidade de Endocrinologia do Desenvolvimento, Faculdade de Medicina da Universidade de São Paulo, SP \\ Danielle Furtado \\ Escola Paulista de Medicina, Universidade Federal de São Paulo, SP \\ Rodolfo Ferreira \\ TCL Soluções, SP \\ Emanuel Carrilho \\ Instituto de Química de São Carlos, Universidade de São Paulo, SP \\ Luciani Carvalho \\ Unidade de Endocrinologia do Desenvolvimento, Faculdade de Medicina da Universidade de São Paulo, SP \\ Nilson Assunção ( $\square$ nilson.assuncao@unifesp.br) \\ Escola Paulista de Medicina, Universidade Federal de São Paulo, SP
}

\section{Research Article}

Keywords: metabolomics, lipidomics, mass-spectrometry, GH, biomarkers

Posted Date: November 10th, 2021

DOI: https://doi.org/10.21203/rs.3.rs-1021675/v1

License: (c) (i) This work is licensed under a Creative Commons Attribution 4.0 International License. Read Full License 


\section{Abstract}

Purpose: The discovery of biomarkers to detect growth hormone deficiency (GHD) and monitor growth hormone replacement therapy (GHRT) remains challenging. Among "omics" technologies used for screening biomarkers, metabolomics stands out as a powerful tool for large-scale identification and quantification of small molecules present in biological matrixes. Metabolomic profiles allow us to infer the phenotypic state; therefore, metabolomics is a great ally in investigating biomarkers and understanding biological processes.

Methods: In this study, global metabolomics ( $\mathrm{N}=39$; range scan 50-600 m/z) and lipidomics ( $\mathrm{N}=36$; range scan 50-1500 $\mathrm{m} / \mathrm{z})$ approaches were performed by high-resolution direct infusion-mass spectrometry. Partial least-square discriminant analysis (PLS-DA) models were used for datadriven diagnosis of GHD and evaluation of GHRT (VIP score >1.5). Cross-validation, permutation test, and ROC curves confirmed the predictive accuracy of PLS-DA models. The features were annotated using accurate mass measurements matched against the metabolomics database.

Results: Data analysis revealed changes in the class of proteinogenic/glycogenic acids, carnitines, $n$-acyl-amines, unsaturated fatty acids, and sulfur amino acids, and pathway analyzes revealed changes in glycerophospholipid metabolism. Regardless of GH therapy, GHD individuals remain with changes in lipids and amino acids compared to healthy control.

Conclusion: GHRT influences the metabolism status of GHD patients in order to compensate for dysregulations caused by GHD. The data has corroborated the action of GHRT and indicated new potential biomarkers for treatment follow-up.

\section{Introduction}

The pituitary gland sometimes referred to as the body's "master gland," controls the production of several hormones that are essential for human development, such as ACTH (adrenocorticotrophic hormone), responsible for the production of cortisol; LH (luteinizing hormone), linked to ovulation or testosterone production; PRL (prolactin), related with breast milk production after childbirth; and GH (growth hormone), responsible for the human growth, including muscular and bone effects [1]. When the pituitary gland fails to produce one or more hormones, it causes congenital hypopituitarism, estimated to affect one in 3000-4000 newborns. Among hypopituitarism disorders, one of the most relevant is the disturbance in GH production, causing severe disruption in infant development [2]. GH plays a fundamental role in postnatal growth, with several biological functions, including intermediate lipid metabolism and homeostasis [3,4]. Consequently, growth hormone deficiency (GHD) is often accompanied by obesity, hyperlipidemia, abnormalities in cardiac structure, and bone mass decrease [5]. Most of the actions of GH are mediated by IGF-1 (insulin-like growth factor 1); therefore, GHD may occur not only by GH deficiency itself but also due to abnormalities in the GH-IGF-1 axis [6].

In adulthood, the GHD condition is a well-known clinical condition caused by genetic factors or acquired injury and leads to abnormal body composition, poor quality of life, dyslipidemia, increased cardiovascular risk, and mortality [7]. These symptoms can be mitigated with growth hormone replacement therapy (GHRT); however, adverse effects are also reported (e.g., insulin resistance, hypertension, fluid retention). Although been a well-define condition, the lack of uniformity of the disease and analytical protocols remains a challenge for the precise diagnosis of GHD $[4,8]$.

For GHD diagnosis, it is necessary to evaluate extensive biochemical and imaging exams, including quantification of IGF1 and insulin-like growth factor binding protein-3 (IGFB3) to assess GH function indirectly. However, only IGF1 or IGFB3 levels alone are neither sensitive nor specific enough to diagnose hypofunction, and it usually is necessary to expose the patients to more stressful and particular tests that induce maximum viable GH secretion by hypophysis (e.g., arginine, clonidine, and hypoglycemia tests)[9-11]. Even though IGF-1 is still considered a biological marker for GHD pathophysiology, tests designed to measure levels of the factor lack precision, specificity, and robustness.

Metabolomics efficiently creates explanatory and individualized physiological panoramas that reflect the phenotypic state [12,13]. Notwithstanding, only a few papers reported the application of omics for GHD studies [14-18]. In a recent review published by this group, SanMartin and Cols (2020) compiled the studies regarding GHD and considered metabolomics an essential and underused tool for the diagnosis of GHD due to differences in the lipid metabolism between the evaluated cohorts (e.g., fatty acids, $\beta$-oxidation, glycerolipids) [19]. In metabolomic studies, liquid chromatography coupled to mass spectrometry (LC-MS) is consecrated; however, with better resolution mass spectrometers, it is possible to enhance a broad metabolic coverage by directly injecting samples into the ionization source [13,20-22]. Moreover, metabolomics can be applied not only for biomarker screening but also for a better understanding of GHD and GHRT, contributing to the growth of individualized medicine, the concept when therapies are provided according to the needs of individuals on a case-by-case basis [19].

This pilot study applied metabolomic and lipidomic profiling by direct infusion mass spectrometry (DIMS) to congenital GHD patients with and without GHRT. We evaluated the metabolism effects of the disease and its treatment with growth hormone replacement therapy, showing significant differences between the groups regarding proteinogenic/glycogenic amino acids, carnitines, n-acyl-amines, unsaturated fatty acids, and sulfur amino acids, as the significant changes in glycerophospholipid metabolism. 


\section{Material And Methods}

\subsection{Study design and settings}

The current paper presents a cross-sectional study design that assessed adult patients with congenital growth hormone deficiency (GHD) treated or not with GHRT compared with healthy adults. Inclusion criteria were as follows: be in adult age; for the patient's group was necessary a preliminary diagnosis of GHD and be in proper compliance with prescribed treatment (at least in the past six months). Exclusion criteria were: active smokers; a diagnosis of diabetes mellitus or arterial hypertension; patients with poor compliance (Figure 1).

The samples were collected in the hypopituitarism outpatient clinic developmental endocrinology unit of the University of São Paulo Medical School (FMUSP). In the clinic, 318 patients were initially diagnosed with congenital hypopituitarism, among which 217 are under active monitoring, 83 patients in childhood and transition, and 134 in adulthood. The endocrinology unit of FMUSP is a national reference for GHD patients, an outcome of several years of intense care for hypopituitarism patients. Considering the rarity of the condition, the clinic holds a significant number of GHD patients in the country. After assessing inclusion and exclusion criteria, 39 patients were included in this pilot study (Figure 1). Amongst 39 GHD patients, 25 were non GHRT (GHDoff), while 14 were undergoing GHRT (GHDon), and 14 individuals were selected as healthy controls (Figure 1).

This paper's primary goal consists of exploring GHD through an exploratory metabolic analysis; therefore, three comparative data on metabolomics and lipidomics were performed (i.e., GHDon versus GHDoff, GHDon versus Healthy Control, GHDoff versus Healthy Control). Ethical committee approval can be accessed at CAAE no. 98372718.7.0000.0068 (University of São Paulo) and CAAE no. 98372718.7.3001.5505 (Federal University of São Paulo) authorization. All patients signed a term of consent for participation in the study.

\subsection{Metabolite and lipid extraction (plasma)}

Ice-immersed blood samples were vortexed for $2 \mathrm{~min}$ in ice, and a $100 \mu \mathrm{L}$-aliquot of the plasma was pipetted into an Eppendorf $\AA^{\circledR}$ tube for processing; $300 \mu \mathrm{L}$ of a methanol/ethanol (1:1 v/v) solution was added to ensure protein precipitation in each tube. Samples were vortexed for 1 min and incubated for $5 \mathrm{~min}$ in an ice bath. Finally, the samples were vortexed for a few seconds and centrifuged at 13,000 rpm (15.7 rcf) for 20 min at $4{ }^{\circ} \mathrm{C}$. The supernatants (hydromethanolic extracts) were stored at $-80{ }^{\circ} \mathrm{C}$ and used for global metabolomics [23].

For the lipidomics approach, $30 \mu \mathrm{L}$ of plasma was transferred to an Eppendorf ${ }^{\circ}$ tube, and $225 \mu \mathrm{L}$ of methanol was added in each tube, followed by vortexing for $10 \mathrm{~s}$. Posteriorly, $445 \mu \mathrm{L}$ of chloroform and $185 \mu \mathrm{L}$ of water were added and stirred for one hour. After separating the organic phase (lipids) from the aqueous phase (primary metabolites), the phase with lipids was dried and stored at $-80{ }^{\circ} \mathrm{C}$. On the day of the analysis, the lipidic extracts were resolubilized in ethyl acetate.

As internal standard (IS), a solution containing ${ }^{15} \mathrm{~N}$ and ${ }^{13} \mathrm{C}$ labeled tryptophan in water with a final concentration of $40 \mu \mathrm{M}$ was prepared, and 20 $\mu \mathrm{L}$ was spiked into each sample. Before direct infusion to the mass spectrometer (DIMS), the samples were filtered using a $0.22 \mu \mathrm{m}$ nylon syringe filter.

\subsection{Quality control samples (QC)}

Quality control samples (QC) were prepared from an aliquot of $20 \mu \mathrm{L}$ of each sample and mixed thoroughly. QCs samples were later processed as a regular sample, following the protocol in section 2.2. One QC analysis was carried out throughout the experimental design every five analyses of the random samples. QC samples were displayed on a PCA plot to assess the stability and performance of the analytical system.

\subsection{Direct infusion-mass spectrometry (DIMS)}

Untargeted metabolomic and lipidomic analyses were performed using a high-resolution Orbitrap ${ }^{\circledR}$ mass spectrometer (Thermo Fisher Scientific). The following conditions were used for the mass spectra acquisition: ion source voltage of $3.5 \mathrm{kV}$ (both ionization modes); HESI source temperature of $60^{\circ} \mathrm{C}$; the capillary temperature of $250{ }^{\circ} \mathrm{C}$; and multiple $\mathrm{m} / \mathrm{z}$ range acquisitions. Mass spectrometric data were acquired by direct infusion at a flow rate of $5 \mu \mathrm{L} \mathrm{min}{ }^{-1}$. The mass spectrum scanning range used in metabolomics ranged from 50 to $600 \mathrm{~m} / z$, and lipidomics ranged from 50 to $1500 \mathrm{~m} / \mathrm{z}$. The samples were analyzed randomly to avoid bias.

\subsection{Data pre-processing and alignment by $\mathrm{m} / \mathrm{z}$ ratio}

The .RAW files from the MS analysis were converted to .csv using the Xcalibur® software (version 3.0.63, Thermo Fisher Scientific). To better understand the pre-processing and data alignment of DIMS analysis, results must consist of a table containing two columns: $m / z$ and intensity, with one $\mathrm{m} / \mathrm{z}$ ion and its respective intensity in each row. Different samples contain different ions and generate distinct $m / z$ columns between samples. For multivariate statistical analysis, one column must remain constant in all samples; in LC-MS data, for example, time is the column used as constant. On DIMS analysis, there is no separation, so time cannot be used as constant. Therefore, it is necessary to align the $m / z$ columns from all samples. 
In this work, we developed a user-friendly software called MassAligner, that creates a column containing all the acquired $m / z$ in all samples but removing redundancies. After that step, it searches in the original table from each sample the intensity of each $m / z$ in the column. Once an $m / z$ is not present in that particular sample, the software input zero as intensity, and thereof, the final result is a worksheet within all the samples, with the same $m / z$ column (i.e., a constant column). MassAligner allows round the decimal cases in the $m / z$ column since high-resolution $m / z$ data requires high computational power.

After alignment, the data was processed using the MetaboAnalyst 5.0 platform (https://www.metaboanalyst.ca/) to normalize and prepare the data for multivariate analysis. This study applied normalization, followed by log transformation and Pareto scaling to transform the data matrix into a Gaussian-type distribution. The MassAligner software and raw data are available in a cloud-based repository (http://dx.doi.org/10.17632/dd3p5tfcd7.1 and http://dx.doi.org/10.17632/8z736ssxdj.1, respectively).

\subsection{Statistical analyses}

Principal component analysis (PCA) was performed to verify the data grouping, trends, variables selection, and/or outliers from the group. For group classification, Partial least squares discriminant analysis (PLS-DA) was carried out on the data. The predictive ability and statistical significance of the PLS-DA model were accessed through cross-validation applying a permutation test with 1000 interactions, and ROC curves for features with Variable importance for the projection (VIP) score $>1.5$ were performed for accuracy verification. Only 36 of the 39 samples were used for data analysis in the lipidomics approach due to experimental problems in three of them. In total, 12 PLS-DA and 4 PCA analyses were performed and are available as supplementary material together with the permutation tests and ROC curves.

\subsection{Putative annotation, enrichment, and pathways analysis}

METLIN, Human Metabolome Database (HMDB), and LIPIDMAPS databases were employed for the putative annotation. A mass error of 5 ppm was set as the maximum tolerable variation from the measured $\mathrm{m} / \mathrm{z}$. Chemical modifications (adducts) in both ionization modes were considered. Enrichment and pathways analysis was performed in MetaboAnalyst (version 5.0).

\section{Results}

\subsection{Anthropometric and clinical data}

Clinical and anthropometric parameters from the patients are presented in Table 1. It is interesting to highlight that among all GHD patients, 12 have isolated GHD diagnosis, i.e., only GHD hormone is unregulated. In contrast, 27 patients have combinate GHD diagnosis, i.e., GHD and other(s) hormones are unregulated. Therefore, due to similar hormonal conditions, some patients utilize levothyroxine, sex steroids, and glucocorticoids. Although the difference in patients under GHD therapy on and off is not statistically significant, the therapy may contribute to the alterations between the GHD and control groups.

Although higher triglycerides and lower HDL levels were observed in the GHDoff group, there was no significant statistical difference between the groups in any classic clinical lipid profile parameter (Table 1). It is important to note that three patients in the GHDoff group were under statin therapy for control of cholesterol levels; nonetheless, once these samples were excluded, the median and statistical significance of the clinical classic lipidic profile between the groups had no alteration; therefore, the three samples were used for the omics studies.

From the anthropometric data, one may notice a significant difference in Body Mass Index (BMI) between GHDoff vs. GHDon, with a higher percentage of eutrophic individuals between control patients and GHDon group; these body characteristics are closely related to the role of GH in the body composition. There is a significant difference in IGF-SD between GHD and controls patient, but no difference between GHDon vs. healthy controls, indicating that the therapy could correct the IGF-1 to normal levels. 
Clinical and anthropometric data.

\begin{tabular}{|c|c|c|c|c|c|}
\hline \multicolumn{5}{|c|}{ GHD patients } & \multirow[t]{2}{*}{ Controls } \\
\hline \multicolumn{2}{|l|}{ Clinical data } & All GHD & GHDon & GHDoff & \\
\hline \multicolumn{2}{|l|}{$\mathbf{N}$} & 39 & 14 & 25 & 14 \\
\hline \multicolumn{2}{|l|}{ Sex (female) } & $23(61 \%)$ & $8(57 \%)$ & $15(60 \%)$ & $7(50 \%)$ \\
\hline \multicolumn{2}{|l|}{ Mean age (years old) } & $38.8( \pm 7.1)$ & $37( \pm 7.2)$ & $39.8( \pm 7.1)$ & $36( \pm 8.62)$ \\
\hline \multicolumn{2}{|l|}{ Mean age at diagnosis (y.o) } & $15.4( \pm 7.2)$ & $15.17( \pm 7.6)$ & $15.6( \pm 6.9)$ & - \\
\hline \multicolumn{2}{|l|}{ Mean final height (m) } & $14.6( \pm 9.1)$ & $21.8( \pm 5.0) \rrbracket$ & $10.6( \pm 8.59)^{\square}$ & - \\
\hline \multicolumn{2}{|l|}{ Mean BMI $\left(\mathrm{kg} \mathrm{m}^{-2}\right)$} & $1.56( \pm 0.12)$ * & $1.59( \pm 0.09)^{¥}$ & $1.55( \pm 0.12) \otimes$ & $1.70( \pm 0.10) * ¥ \rrbracket$ \\
\hline \multicolumn{2}{|l|}{ BMI classification (\%) } & $23.7( \pm 3.47)$ & $21.5( \pm 2.3)^{\otimes}$ & $24,9( \pm 3.8)^{\otimes}$ & $29.3( \pm 7.3)$ \\
\hline \multirow[t]{3}{*}{ BMI classification (\%) } & Slim/eutrophic & 66.7 * & $92.3^{\bigotimes}$ & $56.5^{\bigotimes} \otimes$ & $92,8 * \square$ \\
\hline & Overweight & $25.6^{*}$ & $7,6^{\rrbracket}$ & $30.4^{\llbracket} \rrbracket$ & $0 * \nabla$ \\
\hline & Obese & 7.7 & 0 & 13.1 & 7.2 \\
\hline \multicolumn{2}{|l|}{ Levothyroxine treatment (\%) } & $74.3^{*}$ & $78.5^{¥}$ & $72 \otimes$ & $0 * * \otimes$ \\
\hline \multicolumn{2}{|l|}{ Desmopressin use (\%) } & 7.7 & 7.1 & 8 & 0 \\
\hline \multicolumn{2}{|c|}{ Sexual hormone replacement (\%) } & 61.5 * & $50 *$ & $68 \otimes$ & $0 * ¥ \otimes$ \\
\hline \multicolumn{2}{|l|}{ Glucocorticoid treatment (\%) } & 38.4 * & $42.8^{¥}$ & $36 \otimes$ & $0 * ¥ \otimes$ \\
\hline \multicolumn{2}{|l|}{ Statin use } & 7.7 & 0 & 12 & 0 \\
\hline \multicolumn{2}{|l|}{ rhGH treatment (\%) } & 35.8 * & $100^{\square}$ & $0^{\mathbb{1}}$ & $0 * *$ \\
\hline \multicolumn{2}{|l|}{ IGF-SD } & $-3.50 \mathrm{SD}( \pm 2.25)$ * & $-0.65 \mathrm{SD}( \pm 1.53)^{\mathbb{Q}}$ & $-5.1 \mathrm{SD}( \pm 1.29) \otimes \otimes$ & $0.11 \mathrm{SD}( \pm 0.68) * \otimes$ \\
\hline \multirow{4}{*}{$\begin{array}{l}\text { Classic clinical lipidic profile } \\
\left(\mathrm{Mg} \mathrm{dL}^{-1}\right)\end{array}$} & Total cholesterol & $190 \pm 24.3$ & $184 \pm 20.2$ & $193 \pm 26.5$ & $176 \pm 21.1$ \\
\hline & HDL & $60 \pm 16.1$ & $68 \pm 16.5$ & $56 \pm 14.1$ & $55 \pm 16.1$ \\
\hline & LDL & $110 \pm 22.0$ & $100 \pm 16.5)$ & $116 \pm 22.4$ & $103 \pm 11.8$ \\
\hline & Triglycerides & $92 \pm 33.9$ & $77 \pm 24.9$ & $101 \pm 38.4$ & $81 \pm 24.4$ \\
\hline
\end{tabular}

\subsection{Statistical analysis}

Initially, PCA analysis was applied to the data to search for grouping tendencies and analytical variation; however, no clear group separation could be observed in PCA analysis for metabolomics and lipidomics data. The QCs clustering can establish the robustness of the analytical method in metabolomics approach analysis (Figure 2A). The other PCA figures for analysis of lipidomic and metabolomic approaches are available in the supplementary material in Figure $\mathbf{S} 1$.

Considering that the PCA model could not clearly show the grouping of the samples, PLS-DA, a supervised multivariate analysis, was employed in the data. Figures $2 \mathrm{~B}$ and $2 \mathrm{C}$ report the PLS-DA model and the coefficients that infer the fitting and predictive ability of each PLS-DA. Although the $\mathrm{R}^{2}$ and $\mathrm{Q}^{2}$ metrics indicate the reliability of the generated model, metabolomics and lipidomics PLS-DA analysis were validated by permutation test and area under the curve (AUC) of the ROC curve for assurance of statistical significance and predictive performance of the model. The PLSDA shown in Figure 2C shows the GHDon vs. GHDoff (+ mode) group comparison for the metabolomic approach; Figure 2D and 2E represent the permutation test and roc curve used in the model validation, respectively. In the supplementary material, all the PLS-DA comparison models are presented in Figure $\mathbf{S} 2$ for the metabolomics approach, followed by Figure $\mathbf{3} 3$ with the ROC curves for all the PLS-DA models and the permutation tests in Figure S4. For the lipidomics approach, the PLS-DA models are presented in Figure S5, and the ROC curves and permutation tests in Figure S6 and S7. 
For multivariate exploratory analysis based on the ROC curve, only features with VIP > 1.5 were selected. ROC curves for the comparisons of GHDon vs. GHDoff, GHDon vs. healthy control, and GHDoff vs. healthy control are reported in Figure 3.

After the reliability check of the PLS-DA model, the annotation of the features was performed based on the high-resolution $m / z$ data, based on the set of variables that distinguished the groups and allowed the verification of the similarities in the annotated species, regardless of the GHRT and "omics" approach (Figure 3). The area under the curve (AUC), the 95\% confidence interval, and the $p$-value for each variable putatively annotated from the univariate ROC curve is reported in Table S1.

\subsection{Putative annotation, enrichment, and pathways analysis}

Metabolomics standards initiative (MSI)[24] guidelines and International lipid classification and nomenclature committee (ILCNC)[25] guidelines were adhered to for the annotation of significant metabolites (Table 2). Table 2 reports the database correspondences based on the precise mass within a low mass tolerance ( 5 ppm) corresponding to a single chemical formula; identification numbers (ID) of each annotated feature are available in Table S1 and Table S2.

Metabolomics and lipidomics approach of the GHD group revealed features inherent in the changes caused by GHD in adults and the action of GHTR (Table 2). Twelve PLS-DA models were constructed using two-class input (i.e., GHDon vs. GHDoff; GHDon vs. healthy; GHDoff vs. healthy, both with negative and positive ion mode). 
Table 2

Putative annotations of VIP compounds between GHDon vs. healthy, GHDoff $v s$. healthy, and GHDon vs. GHDoff analyses (VIP score >1.5).

\section{Metabolomics}

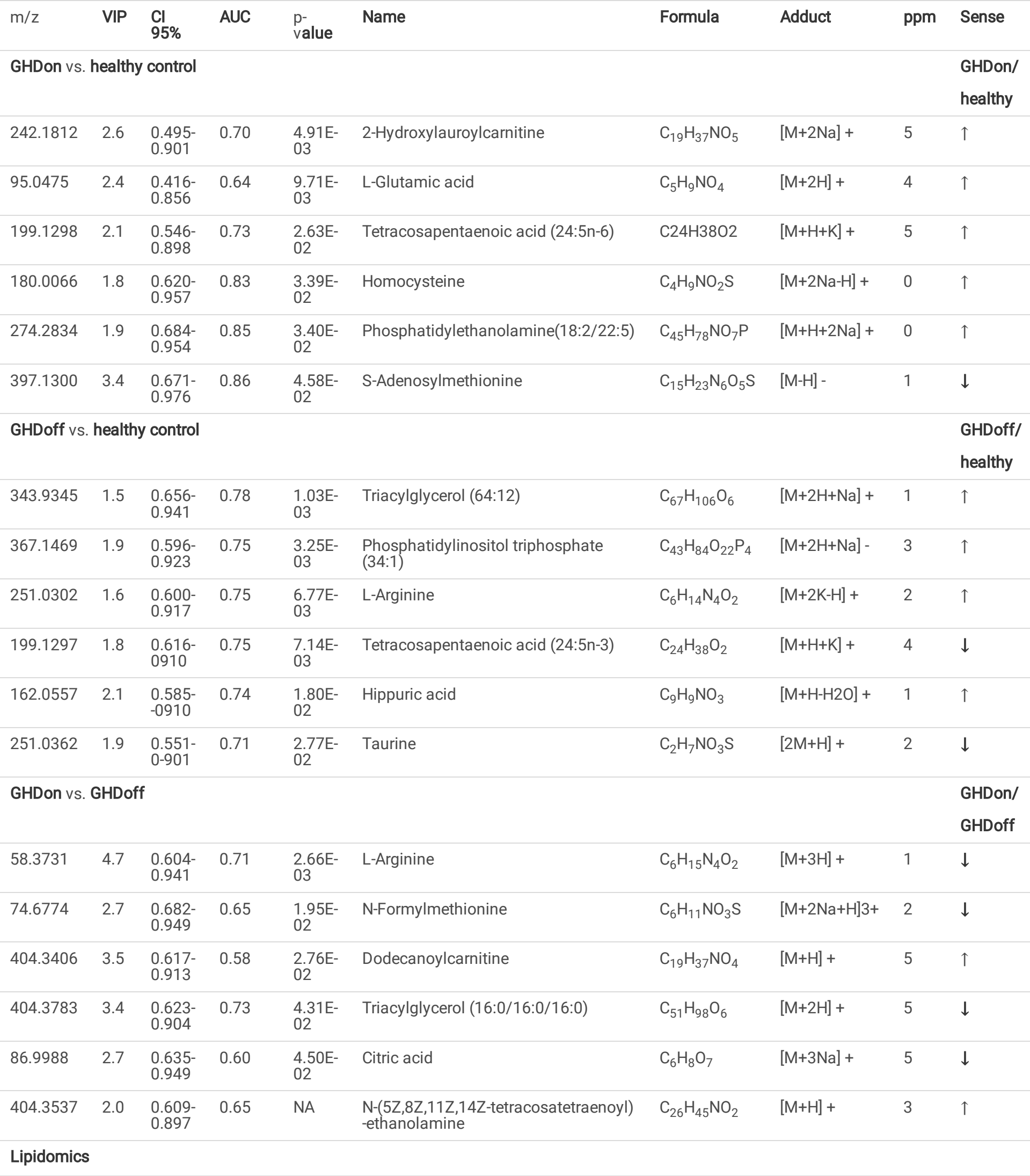

* Putative annotations were assigned using threshold mass measurements (ppm < 5) combined with the HMDB, Metlin, and LipidMaps databases. 


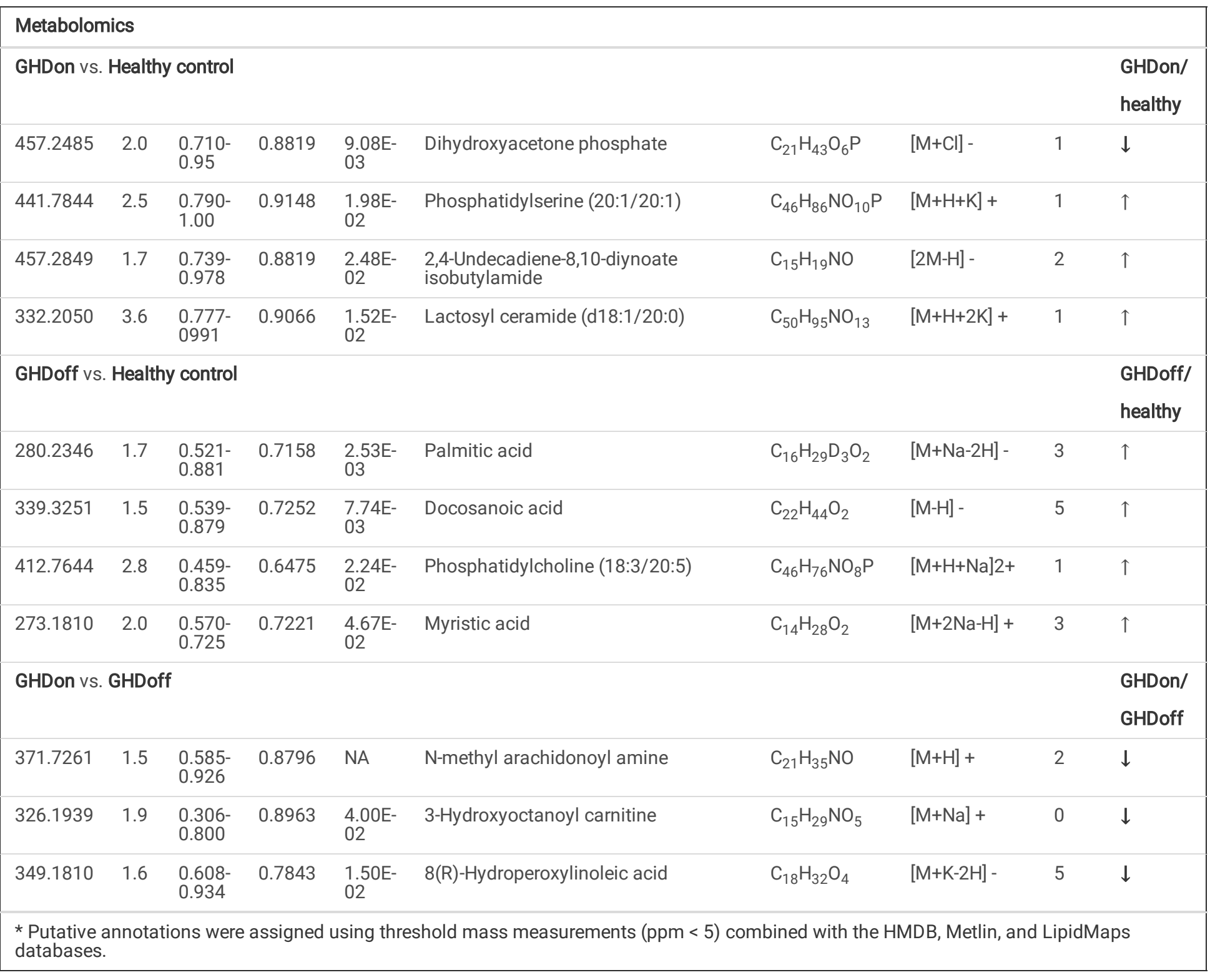

The enrichment analyses showed the main chemical classes of annotated features in metabolomics and lipidomics approaches (Figure 4A and 4B). Pathway analyzes showed metabolic changes linked to GHD (Figure 4A and Figure 4B). For enrichment and path analysis, only the features of comparisons the GHDon vs. control healthy and GHDoff $v s$. healthy control were used to build them. Figure $4 \mathrm{C}$ presents the main classes of altered metabolites and their path according to the therapeutic conditions (i.e., with GHTR and without GHRT).

\section{Discussion}

The IGF-1 as a biomarker did not show differences between GHDon and healthy control (Table 1); however, the metabolomics/lipidomics could predict significantly altered sets of small molecules. Consequently, the use of IGF-1 as a biomarker is again considered reductionist and not very sensitive during adulthood. The low specificity of biomarkers for GHD and the evident change in lipid metabolism led to the application of nontargeted metabolomics/lipidomics in the search for new sources of biomarkers linked to the phenotypic state of GHD (i.e., downstream readouts of cellular signaling, transcriptomic and proteomic changes).

Metabolomics and lipidomics analysis by DIMS showed good coverage of the GHD metabolome; notwithstanding, it is important to highlight those similar results can be achieved computational algorithms in treating DIMS data, then such platforms require previous knowledge in computing programming. For this reason, we developed MassAligner software to be user-friendly, regarding previous programming knowledge. DIMS may be a more practical alternative in large-scale metabolite studies due to lower time consumption in data acquisition, and MassAligner Software guaranteed the accessible Metaboanalyst ${ }^{\circledR}$ platform. 
The PLS-DA model proved to be efficient in the data drive prediction of GHD through the metric values of the cross-validation; prediction coefficients $\left(\mathrm{Q}^{2}\right)$ are in agreement with those suggested in metabolomic studies (i.e., Q2>0.6) (Figure 2) [26]. Permutation test and ROC curve corroborate the observed statistical difference between the sample means and predictive performance of PLS-DA models [27].

Other studies have already demonstrated the potential of metabolomics to elucidate biochemical pathways and unknown metabolites [28,29]. Among the Omics sciences applied in GHD, metabolomics is scarcer than the large-scale molecular study techniques used in molecular biology (i.e., transcriptomics) $[17,18]$. A recent study using single-cell transcriptomics has provided insights into the transcriptional landscape of human pituitary development, which will allow data integration for the development of multi-omics GHD studies [18].

\subsection{Metabolomics approach}

Although the metabolomics sample preparation protocol favors polar metabolites, the glycerophospholipid class produced significant features that classify GHD (Figure 4A and 4B). It is interesting to highlight that the participation of glycerophospholipids can be established from the structural composition of cells, mediation of cellular responses, and precursors of biological effectors [30-32]. Thus, the glycerophospholipid alterations in the GHD population were considered: 1) subsequent action of the change in fatty acids; 2) high oxidative stress coupled with the imbalance of reactive oxygen species can destabilize membranes glycerophospholipids; 3 ) sources of building blocks for biological effectors.

Amino acids also stood out as significant metabolites to differentiate GHD individuals from healthy controls, which directly reflects the ability of metabolomics and its branches to create panoramas of the studied phenotype. The pathways analysis indicated a significant change in the metabolism of the following amino acids: glutamine/glutamate, taurine/hypotaurine, cysteine/methionine, arginine/proline, alanine/serine/glutamate (Figure 4B). Alteration of amino acids metabolism are reflective of the metabolic actions of homeostasis during the lack of $\mathrm{GH}$ as a biological effector so that it can lead to hypoglycemia (i.e., $\mathrm{GH}$ acting as an insulin counter-controller); consequently, these proteinogenic and glycogenic amino acids are redirected to catabolism (e.g., glutamine, arginine, proline, alanine). Amino acids can be obtained from proteolysis of muscle tissues and follow as an energy substrate in GHD [33]; hence, glycolysis/gluconeogenesis and the TCA cycle are intermediary pathways that suffer from substrate alternation.

The change in the pathways of sulfuric amino acids (e.g., taurine/hypotaurine, cysteine /methionine) makes up the need to use the thiol group available in these amino acids for various molecules with biological actions (e.g., Glutathione) [34]. From an endocrinological point of view, this condition may be related to the fact that several biomolecules require the help of sulfotransferase enzymes to perform their metabolic functions effectively (e.g., steroids, bile salts, peptides) [35]. With proteomic analysis and omics data integration, it will be possible to determine which enzymes are involved with oxidative stress in GHD conditions.

Protein synthesis uses arginine in the anabolic process, and glutamine is an essential precursor for arginine biosynthesis [36,37]. The annotation of glutamic acid, cysteinyl-proline peptide, arginine, capreomycidine directly impacted the arginine metabolism in GHDon and GHDoff conditions (Table 2). Oh, et al. (2017) demonstrated that L-arginine promotes cellular proliferation and increases phosphorylation of proteins linked to the MAPK/ERK signaling pathway. The role of L-arginine also includes enhancing the expression of genes responsible for GH and IGHF-1 regulation [36].

Data from the PLS-DA model of GHDon vs. healthy control and GHDoff vs. healthy control, Table 2, shows that regardless of GHRT, there are variables (features or putative metabolites) that remain unregulated in the patients. Upregulation and downregulation from these metabolites, such as tetracosapentaenoic acid ( $m / z$ 199.1297) (Table 2), show that GHRT influences the metabolism status of GHD patients in order to compensate for dysregulations caused by the disease. GHDon vs. GHDoff analysis corroborates the action of GHRT through the projection of unbalanced variables compared to healthy control. Table 02 contains significant annotated vital metabolites associated with amino acid and lipid metabolism, and a list of all noted analytes and their structural information and annotation are available in Table S1. Besides lipid and amino acid changes, bioenergetic metabolism appears to be subsequently affected due to the partial or total absence of GH in homeostatic control (e.g., alterations in TCA, $\beta$-oxidation, glycolysis/gluconeogenesis levels). Recently, Hoffman and Cols (2020) have used metabolomics and transcriptomics analyses in growth hormone-releasing hormone knockout (GHRH-KO) in mice, and the results showed increased transcript levels of mitochondrial amino acid genes; while metabolomics showed that mitochondrial metabolites are differentially regulated in GHRH-KO as well signal of genotype-by-sex interactions [38].

\subsection{Lipidomics approach}

As the metabolomics results suggested the importance of lipids in GHD patients, a lipidomics approach was carried out in the samples. The results from the new approach corroborated with the previous study, indicating a significant change in the glycerophospholipid class (Figure $3 \mathrm{~A}$ and $4 \mathrm{~A}$ ). It was observed that the enrichment analysis revealed important species of lipids that act as biological effectors during the bioenergetic metabolism of GHD, such as acyl-carnitines, glycerolipids, unsaturated fatty acids, and n-acyl-amines. These classes of metabolites proved to be significant regardless of therapeutic approaches in individuals with GHD (Figure 4A). In parallel, metabolomics pointed to similar lipid species, although only the glycerophospholipids were significant in the metabolomics pathway analysis (Figure 4B).

Page 9/16 
Another metabolic difference in GHD patients revealed by the lipidomics approach was the change of fatty acyls metabolism, noted by the putative annotation of lipids such as Palmitic acid; Docosanoic acid; Myristic acid; 8-Hydroperoxylinoleic acid. It must be noted that Fatty Acyls correspond to a "super" lipid class formed by the loss of hydroxyl from the carboxy group of a fatty acid. The synthesis and oxidation of fatty acids are well defined by the enzymes ACC1 and ACC2 that regulate a set of reactions in the cytosol and mitochondria, respectively [39]. The condition of GHD prevents insulin counter-regulation; therefore, there will be greater intensity in fatty acid elongation, which explains one of the parameters that unbalance the metabolism of fatty acids $[33,40]$.

Moreover, acyl-carnitines such as 3-hydroxyoctanoyl carnitine; Linoleoyl carnitine were up-regulated in the GHDoff individuals (Table 2). These unbalanced lipids are participants in biosynthesis and lipid oxidation metabolism, indicating that the absence of GHRT in adulthood affects bioenergetic metabolism [40].

Lipolysis is directly linked to $\mathrm{GH}$ stimuli; however, the absence of GH does not correctly stimulate the use of lipid reserves found in adipocytes to obtain energy and/or intermediate metabolites [41]. At the same time, the proteolysis of muscles becomes a source of energy, which is significant in GHD [42,43]. Treep and Cols (2008) showed that GH therapy stimulates oxidation of lipids originating from skeletal muscle in GHD patients [44]; therefore, $\mathrm{GH}$ is an important parameter that regulates the use of these lipid reserves efficiently, and metabolomic/lipidomic profile could guarantee a better understanding of GHRT.

The lipid changes noted between GHDon vs. healthy control are associated with bioenergetic metabolism (e.g., 2-hydroxydecanedioic acid), communication/signalization (e.g., 8-Hydroperoxylinoleic acid), and N-acyl-amines (e.g., 2,4-Undecadiene-8,10-isobutyl amide) that have a role important in lipid transport [45] (Table 2).

Phosphatidylcholine, phosphatidylserine, phosphatidylglycerol, and phosphatidic acids are up-regulated features in GHD individuals regardless of GHRT (Table 2). Differences between patients on GHRT and healthy controls also remained significant in the work of Abd Rahman (2013).

GHD condition has been shown to limit lipids as an energy source and could subsequently affect other biochemical pathways that use lipids, such as inflammation, cognition, metabolic homeostasis, and transport $[45,46]$. The imbalance in lipid diversity can affect essential unsaturated fatty acids that are precursors or intermediates of biological effectors (i.e., prostaglandins, glycerophospholipids, and post-translation modification (PTM) of proteins with acyl groups regulates various biological processes $[47,48]$.

\section{Conclusion}

The difficulty in finding robust biomarkers for GHD and monitoring GHRT action in adults remains a challenge. The search to understand metabolome in GHD patients aimed to clarify the leading products and/or intermediates in the metabolism of GH deficiency, contributing to elucidating the $\mathrm{GH}$ axis. Several parameters influence changes in the lipid profile; however, further study using metabolomics/lipidomics and data integration with the other omics will be essential to create individualized and explanatory panels on the pathophysiological condition of GHD. Metabolomic and lipidomic approaches showed consistent changes in the GHD metabolome and correlated them with the treatment approach. Notwithstanding, other targeted studies with a more significant number of individuals must be carried out to validate the unregulated lipids and/or amino acids as GHD biomarkers.

\section{Declarations}

\section{Conflict of Interest:}

The authors declare that they have no conflict of interest with this paper.

\section{Ethical Approval:}

Samples used in this research paper were approved by the ethical committee CAAE no. 98372718.7.0000.0068 from the University of São Paulo and CAAE no. 98372718.7.3001.5505 from Federal University of São Paulo. All the methods were carried out in line with Ethical Principles for Medical Research Involving Human Subjects.

\section{Informed Consent:}

All patients provided written informed consent for participation in this study. Only after the written consent, their blood was sampled.

\section{Funding:}

This work was supported by the Fundação de Amparo à Pesquisa do Estado de São Paulo (FAPESP) [grant numbers 2014/50867-3, and 2018/18/02385-0]; the Conselho Nacional de Desenvolvimento Científico e Tecnológico (CNPq) [grant numbers 202424/2017-0, 465389/2014-7, 
and 405130/2018-0]; the Pfizer 2017 ASPIRE Global Endocrine grant \# WI230416; and the Coordenação de Aperfeiçoamento de Pessoal de Nível Superior - Brasil (CAPES) - Finance Code 001.

\section{References}

1. Davis SW, Ellsworth BS, Peréz Millan MI, Gergics P, Schade V, Foyouzi N, Brinkmeier ML, Mortensen AH, Camper SA (2013) Pituitary Gland Development and Disease: From Stem Cell to Hormone Production. Curr. Top. Dev. Biol. https://doi.org/10.1016/B978-0-12-416021-7.000018.

2. Dattani MT (2020) Brook's clinical pediatric endocrinology. Hoboken, NJ.

3. Ranke MB, Wit JM (2018) Growth hormone-past, present and future. Nat. Rev. Endocrinol. https://doi.org/10.1038/nrendo.2018.22.

4. Reed ML, Merriam GR, Kargi AY (2013) Adult growth hormone deficiency - benefits, side effects, and risks of growth hormone replacement. Front. Endocrinol. https://doi.org/10.3389/fendo.2013.00064.

5. Garmes HM, Castillo AR (2019) Insulin signaling in the whole spectrum of GH deficiency, Arch. Endocrin. Metab. https://doi.org/10.20945/2359-3997000000188.

6. Sjögren K, Liu JL, Blad K,Skrtic S, Vidal O, Wallenius V, Leroith D, Törnell J, Isaksson OGP, Jansson JO, Ohlsson C (1999) Liver-derived insulinlike growth factor I (IGF-I) is the principal source of IGF-I in blood but is not required for postnatal body growth in mice. P. Natl. Acad. Sci. U.S.A. https://doi.org/10.1073/pnas.96.12.7088.

7. Shlomo M (2019) Pathogenesis and diagnosis of growth hormone deficiency in adults, N. Engl. J. Med. https://doi.org/10.1056/NEJMra1817346.

8. Biscotto IP, Costa Hong VA, Batista RL, Mendonca BB, Arnhold IJP, Bortolotto LA, Carvalho LRS (2020) Vasculometabolic effects in patients with congenital growth hormone deficiency with and without $\mathrm{GH}$ replacement therapy during adulthood. Pituitary. https://doi.org/10.1007/s11102-020-01099-z.

9. Boguszewski CL (2017) Update on GH therapy in adults. F1000Research. https://doi.org/10.12688/f1000research.12057.1.

10. Mukherjee A, Shalet SM (2009) The value of IGF1 estimation in adults with GH deficiency, Eur. J. Endocrinol. https://doi.org/10.1530/EJE-090247.

11. Blum WF, Alherbish A, Alsagheir A, El Awwa A, Kaplan W, Koledova E, Savage MO, (2018) The growth hormone-insulin-like growth factor-I axis in the diagnosis and treatment of growth disorders. Endocr. Connect. https://doi.org/10.1530/EC-18-0099.

12. Jurowski K, Kochan K, Walczak J, Barańska M, Piekoszewski W, Buszewski B (2017) Analytical Techniques in Lipidomics: State of the Art. Crit. Rev. Anal. Chem. https://doi.org/10.1080/10408347.2017.1310613.

13. Lísa M, Cífková E, Khalikova M, Ovčačíková M, Holčapek M (2017) Lipidomic analysis of biological samples: Comparison of liquid chromatography, supercritical fluid chromatography and direct infusion mass spectrometry methods. J. Chromatogr. A. https://doi.org/10.1016/j.chroma.2017.10.022.

14. Xu R, Zhu, Zhang C, Shen G, Feng J (2019) Metabolomic analysis reveals metabolic characteristics of children with short stature caused by growth hormone deficiency. Clin. Sci. https://doi.org/10.1042/CS20181005.

15. Höybye C, Wahlström E, Tollet-Egnell P, Norstedt G (2014) Metabolomics: a tool for the diagnosis of GH deficiency and for monitoring GH replacement?. Endocr. Connect. https://doi.org/10.1530/ec-14-0098.

16. Abd Rahman S, Schirra HJ, Lichanska AM, Huynh T, Leong GM(2013) Urine metabonomic profiling of a female adolescent with PIT-1 mutation before and during growth hormone therapy: Insights into the metabolic effects of growth hormone. Growth Horm. IGF Res. https://doi.org/10.1016/j.ghir.2012.12.001.

17. Murray PG, Stevens A, De Leonibus C, Koledova E, Chatelain P, Clayton PE (2018) Transcriptomics and machine learning predict diagnosis and severity of growth hormone deficiency. JCI Insight. https://doi.org/10.1172/jci.insight.93247.

18. Zhang S, Cui Y, Ma X, Yong J, Yan L, Yang M, Ren J, Tang F, Wen L, Qiao J (2020) Single-cell transcriptomics identifies divergent developmental lineage trajectories during human pituitary development. Nat. Commun. https://doi.org/10.1038/s41467-020-19012-4.

19. De San-Martin BS, Ferreira VG, Bitencourt MR, Pereira PCG, Carrilho E, Assunção NA, Carvalho LRS (2020) Metabolomics as a potential tool for the diagnosis of growth hormone deficiency (GHD): a review. Arch. Endocrinol. Metab. https://doi.org/10.20945/2359-3997000000300.

20. Moehring T, Scigelova M, Ejsing CS (2016) Essential Lipidomics Experiments using the LTQ Orbitrap Hybrid Mass Spectrometer. Thermo Fisher Scientific. AppNote 367.

21. Haijes HA, Willemsen M, Van Der Ham M, Gerrits J, Pras-Raves ML, Prinsen HCMT, Van Hasselt PM, De Sain-Van der Velden MGM, VerhoevenDuif NM, Jans JJM (2019) Direct infusion based metabolomics identifies metabolic disease in patients' dried blood spots and plasma. Metabolites. https://doi.org/10.3390/metabo9010012.

22. Southam AD, Weber RJM, Engel J, Jones MR, Viant MR (2017) A complete workflow for high-resolution spectral-stitching nanoelectrospray direct-infusion mass-spectrometry-based metabolomics and lipidomics. Nat. Protoc. https://doi.org/10.1038/nprot.2016.156.

Page $11 / 16$ 
23. Dudzik D, Zorawski M, Skotnicki M, Zarzycki W, Kozlowska G, Bibik-Malinowska K, Vallejo M, García A, Barbas C, Ramos MP (2014) Metabolic fingerprint of gestational diabetes mellitus. J. Proteomics. https://doi.org/10.1016/j.jprot.2014.03.025.

24. Sumner LW, Amberg A, Barrett D, Beale MH, Beger R, Daykin CA, Fan TWM, Fiehn O, Goodacre R, Griffin JL, Hankemeier T, Hardy N, Harnly J, Higashi R, Kopka J, Lane AN, Lindon JC, Marriott P, Nicholls AW, Reily MD, Thaden JJ, Viant MR (2007) Proposed minimum reporting standards for chemical analysis: Chemical Analysis Working Group (CAWG) Metabolomics Standards Initiative (MSI). Metabolomics. https://doi.org/10.1007/s11306-007-0082-2.

25. Liebisch G, Vizcaíno JA, Köfeler H, Trötzmüller M, Griffiths WJ, Schmitz G, Spener F, Wakelam MJO (2013) Shorthand notation for lipid structures derived from mass spectrometry. J. Lipid Res. https://doi.org/10.1194/jlr.M033506.

26. Szymańska E, Saccenti E, Smilde AK, Westerhuis JA (2012) Double-check: Validation of diagnostic statistics for PLS-DA models in metabolomics studies. Metabolomics. https://doi.org/10.1007/s11306-011-0330-3.

27. Westerhuis JA, Hoefsloot HCJ, Smit S, Vis DJ, Smilde AK, Velzen EJJ, Duijnhoven JPM, Dorsten FA (2008) Assessment of PLSDA cross validation. Metabolomics. https://doi.org/10.1007/s11306-007-0099-6.

28. Jansen RS, Addie R, Merkx R, Fish A, Mahakena S, Bleijerveld OB, Altelaar M, IJlst L, Wanders RJ, Borst P, Van De Wetering K (2015) N-lactoylamino acids are ubiquitous metabolites that originate from CNDP2-mediated reverse proteolysis of lactate and amino acids. Proc. Natl. Acad. Sci. U. S. A. https://doi.org/10.1073/pnas.1424638112.

29. Pareek V, Tian H, Winograd N, Benkovic SJ (2020) Metabolomics and mass spectrometry imaging reveal channeled de novo purine synthesis in cells. Science. https://doi.org/10.1126/science.aaz6465.

30. Rodriguez-Cuenca S, Pellegrinelli V, Campbell M, Oresic M, Vidal-Puig A (2017) Sphingolipids and glycerophospholipids - The "ying and yang" of lipotoxicity in metabolic diseases. Prog. Lipid Res. https://doi.org/10.1016/j.plipres.2017.01.002.

31. Schmitz G, Ruebsaamen K (2010) Metabolism and atherogenic disease association of lysophosphatidylcholine. Atherosclerosis. https://doi.org/10.1016/j.atherosclerosis.2009.05.029.

32. Croze ML, Soulage CO (2013) Potential role and therapeutic interests of myo-inositol in metabolic diseases. Biochimie. https://doi.org/10.1016/j.biochi.2013.05.011.

33. Moøller N, Joørgensen JOL (2009) Effects of growth hormone on glucose, lipid, and protein metabolism in human subjects. Endocr. Rev. https://doi.org/10.1210/er.2008-0027.

34. Courtney-Martin G, Pencharz PB (2016) Sulfur Amino Acids Metabolism From Protein Synthesis to Glutathione. Elsevier Inc. https://doi.org/10.1016/b978-0-12-802167-5.00019-0.

35. Mueller JW, Gilligan LC, Idkowiak J, Arlt W, Foster P (2015) The regulation of steroid action by sulfation and desulfation, Endocr. Rev. https://doi.org/10.1210/er.2015-1036.

36. Oh HS, Oh SK, Lee JS, Wu C, Lee SJ (2017) Effects of I-arginine on growth hormone and insulin-like growth factor 1. Food Sci. Biotechnol. https://doi.org/10.1007/s10068-017-0236-6.

37. Morris SM (2007) Arginine Metabolism: Boundaries of Our Knowledge. J. Nutr. https://doi.org/10.1093/jn/137.6.1602s.

38. Hoffman JM, Poonawalla A, Icyuz M, Swindell WR, Wilson L, Barnes S, Sun LY (2020) Transcriptomic and metabolomic profiling of long-lived growth hormone releasing hormone knockout mice: Evidence for altered mitochondrial function and amino acid metabolism. Aging. https://doi.org/10.18632/aging.102822.

39. Wakil SJ, Abu-Elheiga LA (2009) Fatty acid metabolism: Target for metabolic syndrome. J. Lipid Res. https://doi.org/10.1194/jlr.R800079JLR200.

40. Kim S, Park MJ (2017) Effects of growth hormone on glucose metabolism and insulin resistance in human. Ann. Pediatr. Endocrinol. Metab. https://doi.org/10.6065/apem.2017.22.3.145

41. Kovacs P, Stumvoll M (2005) Fatty acids and insulin resistance in muscle and liver. Best Pract. Res. Cl. End. https://doi.org/10.1016/j.beem.2005.07.003.

42. Sakharova AA, Horowitz, Surya S, Goldenberg N, Harber MP, Symons K, Barkan A (2008) Role of growth hormone in regulating lipolysis, proteolysis, and hepatic glucose production during fasting. J. Clin. Endocr. Metab. https://doi.org/10.1210/jc.2008-0079.

43. Biagetti B, Simó R (2021) Gh/igf-1 abnormalities and muscle impairment: From basic research to clinical practice, Int. J. Mol. Sci. https://doi.org/10.3390/ijms22010415.

44. Trepp R, Flück M, Stettler C, Boesch C, Ith M, Kreis R, Hoppeler H, Howald H, Schmid JP, Diem P, Christ ER (2008) Effect of GH on human skeletal muscle lipid metabolism in GH deficiency. Am. J. Physiol. - Endoc. Metab. https://doi.org/10.1152/ajpendo.00010.2008.

45. Meijerink J, Balvers M, Witkamp R (2013) N-acyl amines of docosahexaenoic acid and other n-3 polyunsaturated fatty acids - From fishy endocannabinoids to potential leads. Br. J. Pharmacol. https://doi.org/10.1111/bph.12030.

46. Battista N, Bari M, Bisogno T (2019) N-Acyl amino acids: Metabolism, molecular targets, and role in biological processes. Biomolecules. https://doi.org/10.3390/biom9120822.

Page $12 / 16$ 
47. Hishikawa D, Valentine WJ, lizuka-Hishikawa Y, Shindou H, Shimizu T (2017) Metabolism and functions of docosahexaenoic acid-containing membrane glycerophospholipids. FEBS. Lett. https://doi.org/10.1002/1873-3468.12825.

48. Law SH, Chan ML, Marathe GK, Parveen F, Chen CH, Ke LY (2019) An updated review of lysophosphatidylcholine metabolism in human diseases. Int. J. Mol. Sci. https://doi.org/10.3390/ijms20051149.

\section{Figures}

\section{Casuistry: selected patients}

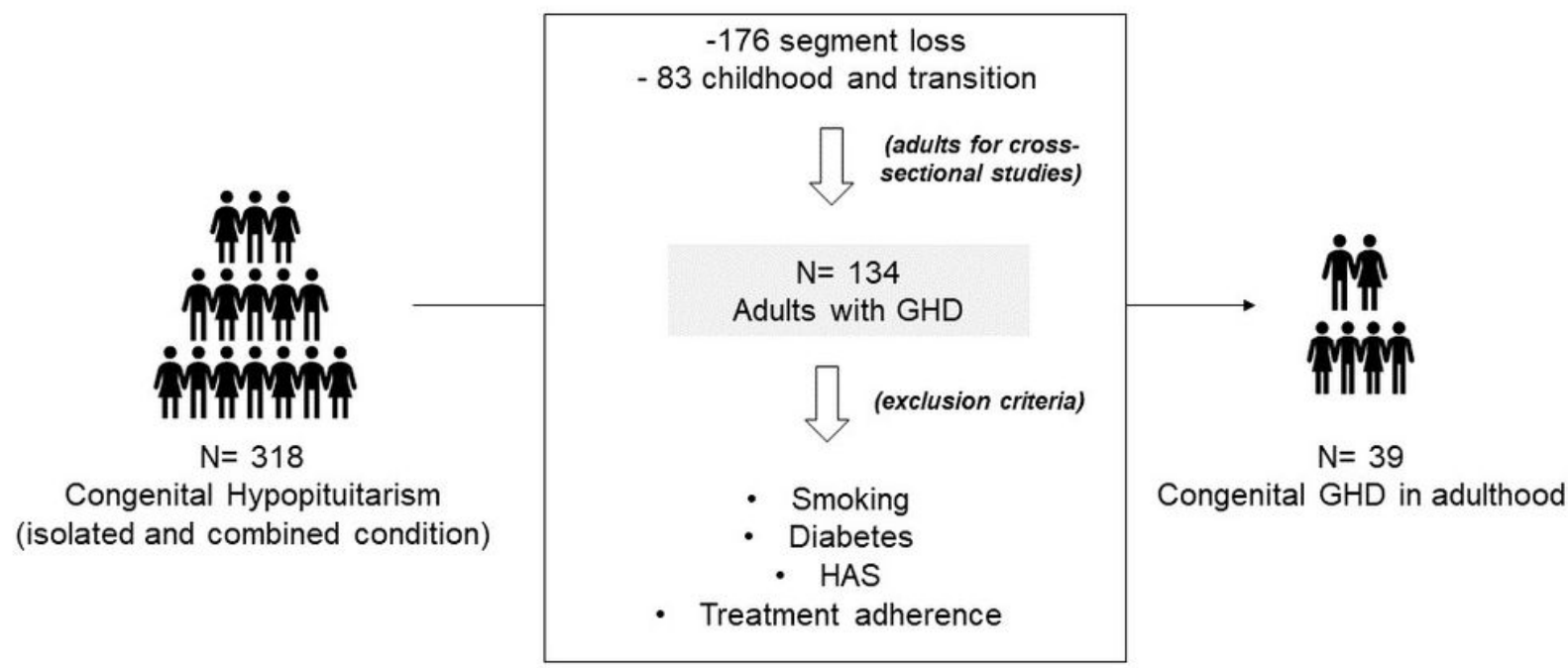

Hypopituitarism Outpatient Clinic /Developmental Endocrinology Unit /University of São Paulo - Medical School

Figure 1

Selection of patients for the metabolomic and lipidomic study of GHD. 


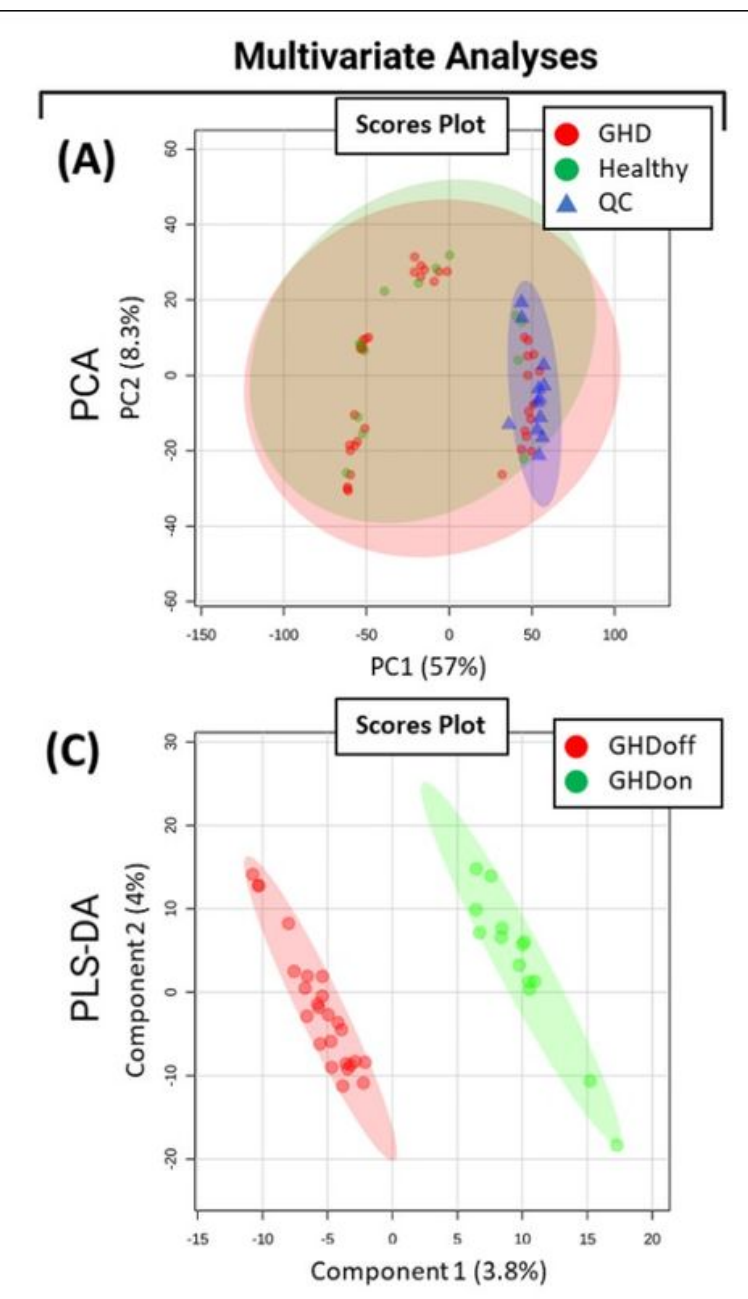

\section{Predicitive Accuracy (PLS-DA model)}

(B)

Cross-Validation

\begin{tabular}{c|l|c|c|c|c}
\hline \multirow{2}{*}{ Approach } & \multirow{2}{*}{ PLS-DA Moldel } & \multicolumn{2}{|c|}{$(+)$ Mode } & \multicolumn{2}{c}{$(-)$ Mode } \\
\cline { 3 - 6 } & & $\mathrm{R}^{2}$ & $\mathrm{Q}^{2}$ & $\mathrm{R}^{2}$ & $\mathrm{Q}^{2}$ \\
\hline \multirow{4}{*}{ Metabolomics } & GHDon vs GHDoff & 0.96 & 0.71 & 0.90 & 0.59 \\
\cline { 2 - 6 } & GHDon vs Healthy & 0.87 & 068 & 0.99 & 0.62 \\
\cline { 2 - 6 } & GHDoff vs Healthy & 0.91 & 0.80 & 0.99 & 0.84 \\
\hline \multirow{4}{*}{ Lipidomics } & GHDon vs GHDoff & 0.94 & 0.78 & 0.99 & 0.85 \\
\cline { 2 - 6 } & GHDon vs Healthy & 0.88 & 0.69 & 0.89 & 0.63 \\
\cline { 2 - 6 } & GHDoff vs Healthy & 0.95 & 0.76 & 0.98 & 0.90 \\
\hline
\end{tabular}
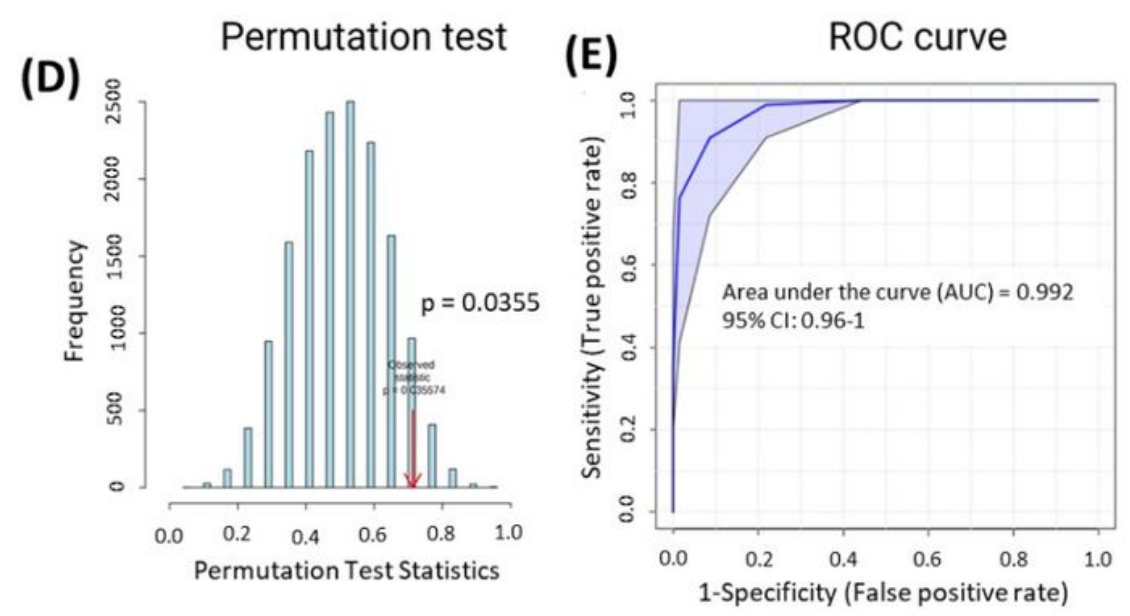

Figure 2

A) PCA model showed the QC clustering in metabolomics approach (positive ion mode); B) fit (R2) and prediction (Q2) parameters of PLS-DA models; C) PLS-DA model in data drive prediction GHD features in GHDon vs. GHDon individuals (positive ion mode); D) observed distribution by permutation test in GHDon vs. GHDoff lipidomics analysis (positive ion mode); E) ROC curve test indicated the accuracy of GHDon vs. GHDoff data matrix (positive ion mode). 
(A) GHDon vs GHDoff

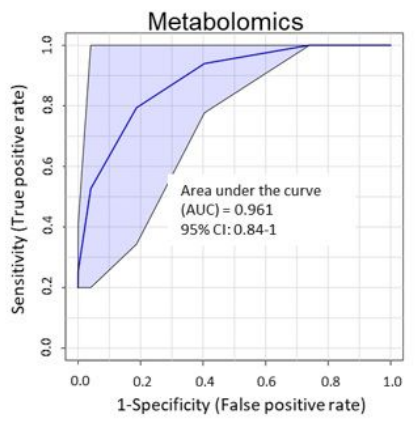

(B) GHDon vs Healthy Control

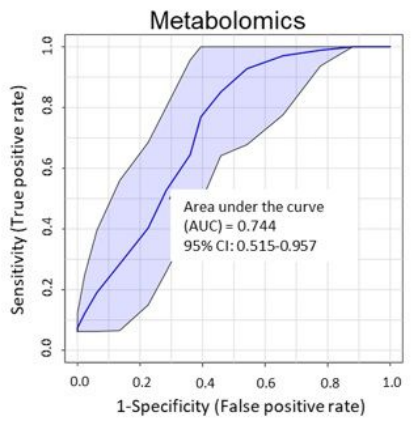

(C) GHDoff vs Healthy Control

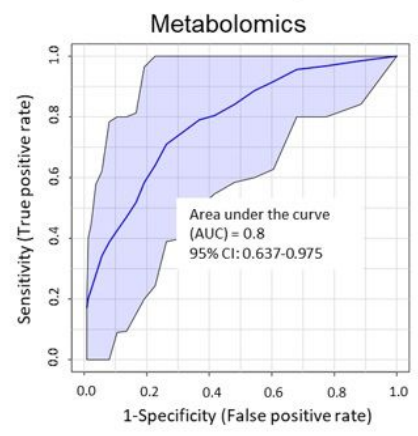

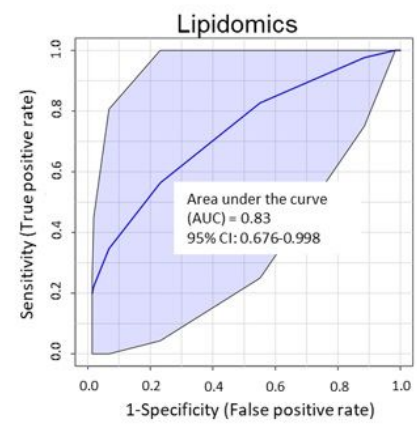
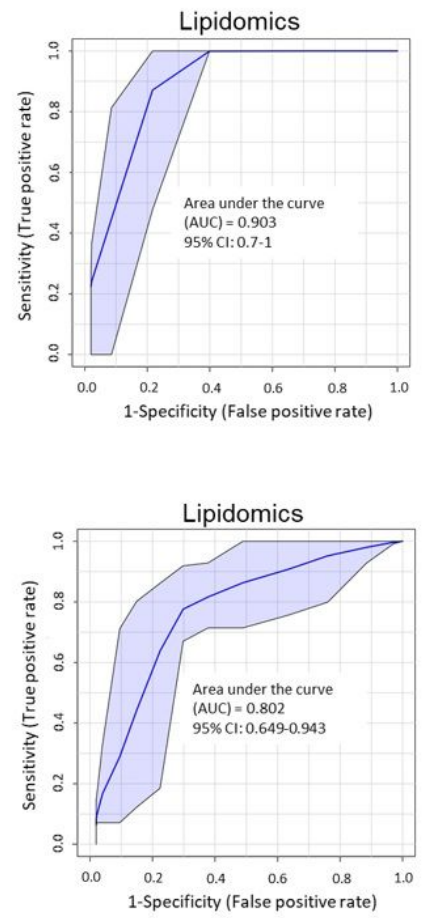

Figure 3

ROC curves for metabolomic and lipidomic data for different group comparisons, based on the PLS-DA algorithm using features with VIP Score > 1.5. A) GHDon vs. GHDoff comparison; B) GHDon vs. Healthy control comparison; and C) GHDoff vs. Healthy control comparison. 
(A) Pathways Analyses

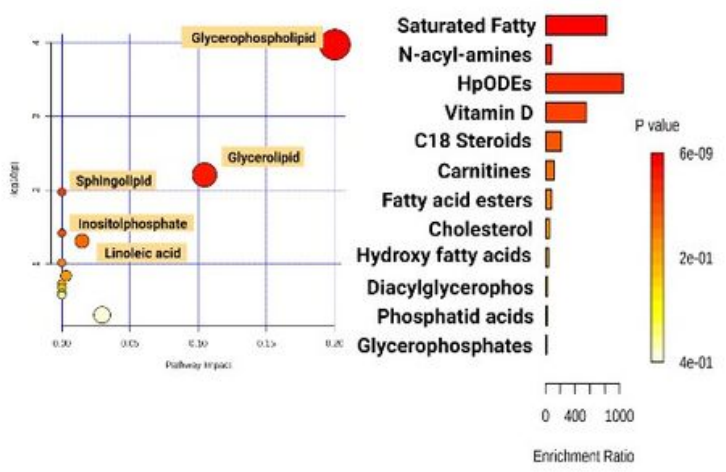

(B)

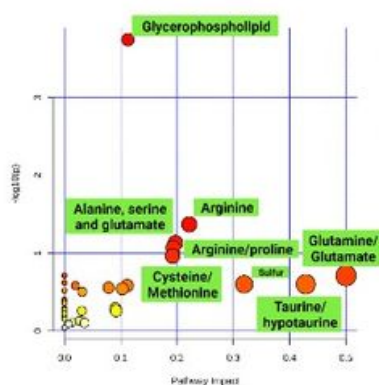

Enrichment Analyses

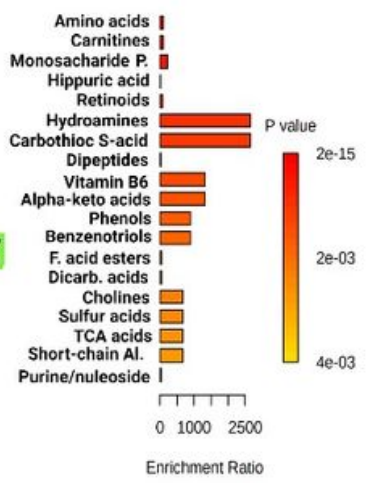

(C)
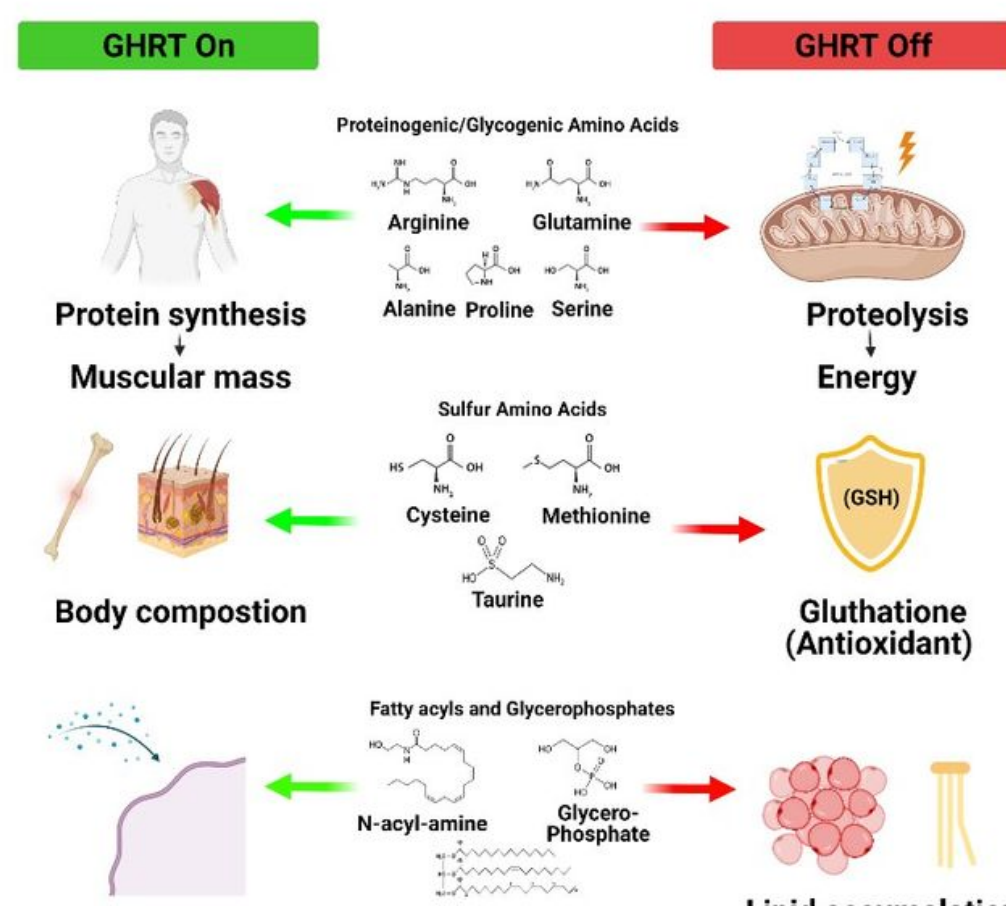

Fatty acyls and Glycerophosphates

Fluidity of lipid bilayer and cell signalization

\section{Figure 4}

A) Pathways and enrichment analyses with annotated features by lipidomics approach. B) Pathways and enrichment analyses with annotated features by metabolomics approach; C) Overview of altered significant metabolites and their impacts on the metabolism of patients with GHRT and without GHRT.

\section{Supplementary Files}

This is a list of supplementary files associated with this preprint. Click to download.

- Supplementary.docx 